\title{
Microbiologically active Mannich bases derived from 1,2,4-triazoles. The effect of C-5 substituent on antibacterial activity
}

\author{
Tomasz Plech • Monika Wujec • Magdalena Majewska • \\ Urszula Kosikowska • Anna Malm
}

Received: 17 March 2012/ Accepted: 14 September 2012/Published online: 29 September 2012

(C) The Author(s) 2012. This article is published with open access at Springerlink.com

\begin{abstract}
Our research proved that chemical character of the C-5 substituent significantly determines the antibacterial activity of the Mannich bases derived from 4,5disubstituted 1,2,4-triazole-3-thiones. This activity was considerably increased by an introduction of a chlorine atom to the phenyl ring. The obtained compounds were particularly active against opportunistic bacteria (Bacillus subtilis and Bacillus cereus). The antibacterial activity of some Mannich bases was similar or higher than the activity of commonly used antibiotics such as ampicillin and cefuroxime.
\end{abstract}

Keywords Aminomethylation · Five-membered ring · Opportunistic bacteria $\cdot$ MRSA

\section{Introduction}

Excessive and uncontrolled intake of antibiotics resulted in a selection of bacterial strains resistant to commonly used drugs. Recently, the world has been focused on the

Study results were presented in part at the Eighth Multidisciplinary Conference on Drug Research, Rawa Mazowiecka, Poland (June, 2012).

T. Plech $(\bowtie) \cdot$ M. Wujec $\cdot$ M. Majewska

Department of Organic Chemistry, Faculty of Pharmacy, Medical University, Chodzki 4A, 20-093 Lublin, Poland

e-mail: tomasz.plech@umlub.pl

U. Kosikowska $\cdot$ A. Malm

Department of Pharmaceutical Microbiology, Faculty of Pharmacy, Medical University, Chodzki 1, 20-093 Lublin, Poland appearance of the so-called super resistant NDM-1 gene (Yong et al., 2009; Rolain et al., 2010) which spreads via DNA segments called plasmids. In the view of growing bacterial drug-resistance, the search of chemical substances which can efficiently treat infections caused by this type of bacteria seems to be necessary.

The Mannich reaction is known to be very useful for the synthesis of antibacterial compounds. This reaction makes it possible to introduce amine fragment into the different chemical scaffolds which can increase the affinity of the obtained molecule toward appropriate molecular target. 1,2,4-Triazole-3-thione derivatives known for their antibacterial activity (Turan-Zitouni et al., 2005; Eswaran et al., 2009; Shafiee et al., 2002) were used by many researchers as substrates for the Mannich reaction. The obtained aminomethyl derivatives included both compounds which acted stronger than their N2-unsubstituted predecessors (Isloor et al., 2009; Ashok et al., 2007; Bayrak et al., 2009a), as well as significantly less active compounds (Bayrak et al., 2009b; Almajan et al., 2009). In our previous studies we proved that the presence of the 4-bromophenyl moiety in the N-4 position benefited the antibacterial activity of 4,5-disubstituted 1,2,4-triazole-3-thione derivatives (Plech et al., 2011a, b). Further research also indicated that the activity of this type of Mannich bases decreases with the increased volume of substituent in the N2 position (Plech et al., 2011b). The goal of current research was to analyze the impact of the substituent in the C-5 position on the antibacterial activity of obtained compounds. First of all, it has been decided to examine if, and to what degree, the strength of the new derivatives' activity changes after introducing a chlorine atom to the phenyl ring. Also, the disparities in the activity of appropriate ortho-, meta-, and para- derivatives were analyzed. 


\section{Results and discussion}

Chemistry

Scheme 1 shows subsequent stages of the synthesis. The substrates for the syntheses included commercially available hydrazides (1-3). Appropriate thiosemicarbazide derivatives (4-6) were obtained from the reaction of the hydrazides (1-3) with 4-bromophenyl isothiocyanate using the method described earlier (Plech et al., 2011a). The reaction carried out in the anhydrous ethanol medium lasted $5 \mathrm{~min}$. Spectral and physicochemical properties of the derivatives 4-6 were given elsewhere (Li et al., 2001; Oruç et al., 2004). The cyclization of compounds 4-6 in the presence of sodium hydroxide resulted in the formation of 4-(4-bromophenyl)-5substituted-2,4-dihydro-3H-1,2,4-triazole-3-thiones (7-9). Generally, the 1,2,4-triazole-3-thione derivatives may exist in two tautomeric forms: thione $(\mathrm{C}=\mathrm{S})$ and thiole $(\mathrm{C}-\mathrm{SH})$. Our earlier studies showed that the thione tautomer is energetically favored (Wujec et al., 2007). The IR spectra of compounds 7-9 showed the absorption bands at $3,437-3,411 \mathrm{~cm}^{-1}$ and $1,331-1,328 \mathrm{~cm}^{-1}$, indicating the presence of $\mathrm{NH}$ and $\mathrm{C}=\mathrm{S}$ groups, respectively. In the ${ }^{1} \mathrm{H}$ NMR spectra, NH proton resonated as a singlet at $\sim 14 \mathrm{ppm}$. Crystallographic data (unpublished results) also confirm the existence of the mentioned compounds as the $\mathrm{C}=\mathrm{S}$ tautomers.

The Mannich reaction was carried out in mild conditions; it was quick (30 min) and efficient (yields: 76-87\%). The structure and purity of the products (10-21) was confirmed using ${ }^{1} \mathrm{H}-\mathrm{NMR},{ }^{13} \mathrm{C}-\mathrm{NMR}$ (for compound 20), and IR spectra as well as elemental analysis. The ${ }^{1} \mathrm{H}-\mathrm{NMR}$ spectra showed characteristic signals which indicated the presence of aminomethyl fragment. Two protons of the N2- $\mathrm{CH}_{2}-$ group resonated as a singlet in the range of 5.22-5.34 ppm, while the signals of the amine residues were visible at 1.20-3.76 ppm. In addition to this, peaks characteristic for para-substituted phenyl rings were visible in the area typical for aromatic protons. The IR spectra also confirmed the suggested structure of the Mannich bases (10-21).

\section{Antibacterial screening}

The antibacterial activity of compounds 10-21 was determined for Gram-positive and Gram-negative bacteria. The growth of Gram-negative bacteria (Escherichia coli ATCC 25922, Klebsiella pneumoniae ATCC 13883, Proteus mirabilis ATCC 12453, and Pseudomonas aeruginosa ATCC 9027) was not inhibited by any of the compounds. Therefore, Table 1 shows the Mannich bases activity only
Scheme 1 Synthetic route to target compounds 10-21.

Reagents and conditions: $a \mathrm{EtOH}$, reflux, $5 \mathrm{~min} ; b 2 \%$ $\mathrm{NaOH}$, reflux, $2 \mathrm{~h}$; $c$ HCHO, amine, EtOH, 30 min

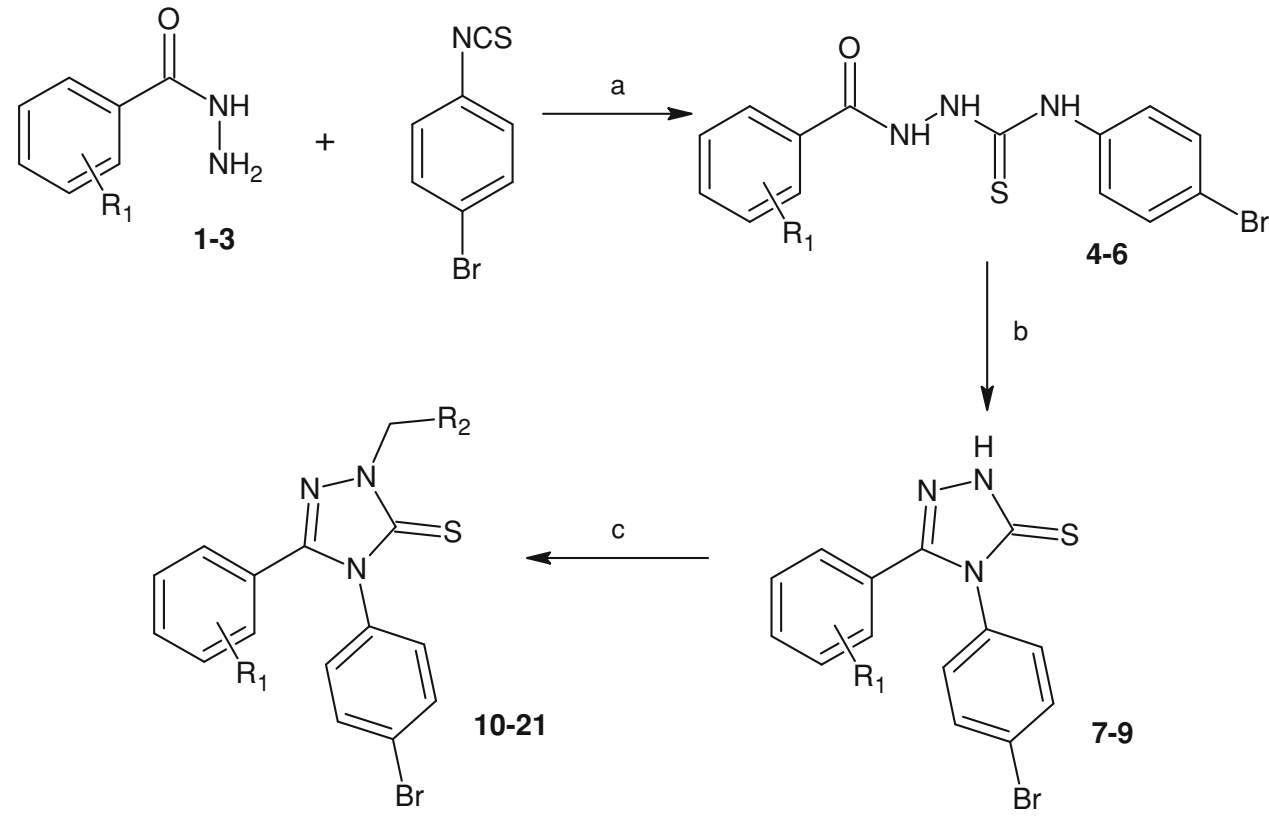

$\mathrm{R}_{1}=\mathrm{H}, 2-\mathrm{Cl}, 4-\mathrm{Cl}$<smiles>[R][CH-]N(C)CC</smiles><smiles>CN1CCCC1</smiles>

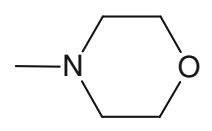


Fig. 1 Chemical structures of compounds 22-25<smiles>[R]Cn1nc(-c2cccc(Cl)c2)n(-c2ccc(Br)cc2)c1=S</smiles><smiles>CCN(CC)CC</smiles><smiles>[Z20]P[PH2+]CN1CCCCC1</smiles>

23. $\mathrm{R}=$<smiles>CN1CCCC1</smiles>

25. $\mathrm{R}$<smiles>P#CN1CCOCC1</smiles>

Table 1 In vitro antibacterial screening of compounds 10-25 (MICs and MBCs are given in $\mu \mathrm{g} \mathrm{ml}^{-1}$ )

\begin{tabular}{|c|c|c|c|c|c|c|c|c|c|c|c|c|}
\hline \multirow[t]{2}{*}{ Compounds } & \multicolumn{2}{|c|}{$\begin{array}{l}\text { S. aureus ATCC } \\
25923\end{array}$} & \multicolumn{2}{|c|}{$\begin{array}{l}\text { S. aureus } \\
\text { (MRSA) }\end{array}$} & \multicolumn{2}{|c|}{$\begin{array}{l}\text { S. epidermidis ATCC } \\
12228\end{array}$} & \multicolumn{2}{|c|}{$\begin{array}{l}\text { B. subtilis ATCC } \\
6633\end{array}$} & \multicolumn{2}{|c|}{$\begin{array}{l}\text { B. cereus ATCC } \\
10876\end{array}$} & \multicolumn{2}{|c|}{$\begin{array}{l}\text { M. luteus ATCC } \\
10240\end{array}$} \\
\hline & MIC & $\mathrm{MBC}$ & MIC & $\mathrm{MBC}$ & MIC & $\mathrm{MBC}$ & MIC & $\mathrm{MBC}$ & MIC & $\mathrm{MBC}$ & MIC & $\mathrm{MBC}$ \\
\hline 10 & 1,000 & $*$ & 1,000 & $*$ & 1,000 & $*$ & 1,000 & $*$ & 1,000 & $*$ & 1,000 & $*$ \\
\hline 11 & 1,000 & $*$ & 1,000 & $*$ & 1,000 & $*$ & 500 & 1,000 & 1,000 & $*$ & 500 & 1,000 \\
\hline 12 & 1,000 & $*$ & 1,000 & $*$ & 1,000 & $*$ & 1,000 & $*$ & 1,000 & $*$ & 500 & 1,000 \\
\hline 13 & 1,000 & $*$ & 1,000 & $*$ & 1,000 & $*$ & 1,000 & $*$ & 1,000 & $*$ & 250 & 1,000 \\
\hline 14 & 62.5 & $*$ & 125 & $*$ & 62.5 & $*$ & 31.25 & 62.5 & 62.5 & $*$ & 62.5 & 250 \\
\hline 15 & 31.25 & 500 & 125 & 500 & 31.25 & 500 & 15.63 & 125 & 62.5 & $*$ & 62.5 & 1,000 \\
\hline 16 & 500 & $*$ & 500 & $*$ & 1,000 & $*$ & 250 & $*$ & 1,000 & $*$ & 500 & $*$ \\
\hline 17 & 125 & 500 & 250 & 500 & 125 & 500 & 125 & 250 & 125 & $*$ & 62.5 & 250 \\
\hline 18 & 31.25 & 250 & 62.5 & 250 & 31.25 & 250 & 31.25 & 500 & 31.25 & 250 & 15.63 & 62.5 \\
\hline 19 & 31.25 & 500 & 62.5 & 1,000 & 31.25 & 1,000 & 62.5 & 125 & 31.25 & $*$ & 7.81 & 250 \\
\hline 20 & 1,000 & $*$ & 1,000 & $*$ & 1,000 & $*$ & 1,000 & $*$ & 1,000 & $*$ & 1,000 & $*$ \\
\hline 21 & 62.5 & 1,000 & 125 & $*$ & 62.5 & 1,000 & 125 & 125 & 62.5 & $*$ & 62.5 & $*$ \\
\hline $22^{\mathrm{a}}$ & 31.25 & $*$ & - & - & 62.5 & $*$ & 62.5 & 500 & 62.5 & $*$ & 31.25 & 500 \\
\hline $23^{\mathrm{b}}$ & 31.25 & $*$ & - & - & 250 & * & 62.5 & 500 & 62.5 & * & 62.5 & $*$ \\
\hline $24^{\mathrm{a}}$ & 31.25 & $*$ & - & - & 62.5 & $*$ & 62.5 & 1,000 & 62.5 & 1,000 & 31.25 & $*$ \\
\hline $25^{\mathrm{a}}$ & 31.25 & $*$ & - & - & 125 & $*$ & 62.5 & 1,000 & 62.5 & $*$ & 31.25 & 500 \\
\hline Ampicillin & - & - & - & - & - & - & - & - & 62.5 & - & - & - \\
\hline Cefuroxime & 0.49 & - & - & - & 0.24 & - & 15.63 & - & 31.25 & - & 0.98 & - \\
\hline Vancomycin & - & - & 0.98 & 3.91 & - & - & - & - & - & - & - & - \\
\hline
\end{tabular}

- not determined, * MIC or MBC values higher than $1,000 \mu \mathrm{g} \mathrm{ml}^{-1}$

a Data derived from Plech et al. (2011b)

b Data derived from Plech et al. (2011a)

for five investigated Gram-positive bacterial strains. The activity toward the pathogenic Staphylococcus aureus strains was moderate. Minimum concentrations which inhibited the growth of $S$. aureus ATCC 25923 ranged to $31.25 \mu \mathrm{g} \mathrm{ml}^{-1}(\mathbf{1 5}, \mathbf{1 8}, \mathbf{1 9})$, and the most active toward methicillin-resistant (MRSA) strain were derivatives with diethylaminomethyl (18) and pyrrolidinylmethyl (19) substituents. In both cases, the MIC values equaled $62.5 \mu \mathrm{g} \mathrm{ml}^{-1}$. Opportunistic (relatively pathogenic) bacteria was by far more sensitive to the newly obtained compounds. In the case of Bacillus cereus ATCC 10876, the activity of three derivatives $(\mathbf{1 4}, \mathbf{1 5}, \mathbf{2 1})$ was similar to the activity of ampicillin, and the activity of another two derivatives $(\mathbf{1 8}, \mathbf{1 9})$ was twice as strong. Moreover, the antibacterial activity of the compound with the N2-pyrrolidinylmethyl fragment (15) toward Bacillus subtilis ATCC 6633 was as strong as cefuroxime's; as far as Micrococcus luteus ATCC 10240 is concerned, the most active compound was the derivative of 4-(4-bromophenyl)-5-(4chlorophenyl)-2,4-dihydro-3H-1,2,4-triazole-3-thione with pyrrolidinylmethyl substituent $\left(\mathbf{1 9}, \mathrm{MIC}=7.81 \mu \mathrm{g} \mathrm{ml}^{-1}\right)$. Based on the MBC/MIC ratio it might be concluded that 
the obtained compounds do show bactericidal (MBC/ MIC $\leq 4$ ) or bacteriostatic (MBC/MIC $>4$ ) activity. This may suggest a presence of more than one mechanism of action for these derivatives.

In order to analyze the impact of the type of substituent in the $\mathrm{C}-5$ position on the antibacterial activity, derivatives including phenyl (10-13), 2-chlorophenyl (14-17), and 4-chlorophenyl (18-21) rings were obtained. In order to ensure more comprehensive analysis, the discussion also considered the compounds with 3-chlorophenyl fragment (22-25) (Fig. 1) - synthesized and described by us recently (Plech et al., 2011a, b). Regardless of the type of aminomethyl substituent in the N2 position, none of the C5phenyl derivatives showed antibacterial activity which would be worth noticing. The activity of the obtained Mannich bases was significantly increased only after an electron-withdrawing chlorine atom had been introduced to the phenyl ring. Interesting conclusions can be drawn when comparing the activity of appropriate ortho-, meta-, and para- analogs. Balanced activity toward all analyzed Gram-positive bacteria was characteristic for compounds with 3-chlorophenyl fragment, regardless of the type of substituent in the $\mathrm{N} 2$ position. While the activity of orthoand para- analogs depended largely on the type of aminomethyl fragment. It is worth mentioning that compounds with pentatomic (apart from hydrogen atoms) amine substituent in the N2 position (pyrrolidine and diethylamine) were microbiologically more active than appropriate compounds with six-membered substituents (piperidine and morpholine). Also, the disparity between the activities of piperidinyl and morpholinyl derivatives shows that the oxygen atom in the morpholine molecule is important for the binding with a potential molecular target. This is probably caused by the fact that the oxygen atom can participate in the formation of hydrogen bonds in the drugtarget site.

\section{Conclusions}

Our research showed that chemical character of the C-5 substituent significantly determines the antibacterial activity of the N2-aminomethyl derivatives of the 1,2,4triazole. This activity can be considerably increased by an introduction of an electron-withdrawing chlorine atom to the phenyl ring in the C-5 position. In addition to this, the number of atoms which form the aminomethyl substituent seems to be important. The activity of the obtained Mannich bases was particularly strong toward opportunistic bacteria. The antibacterial activity of some compounds was similar or higher than the activity of commonly used antibiotics such as ampicillin and cefuroxime.

\section{Experimental}

General comments

All reagents and solvents were purchased from Alfa Aesar (Ward Hill, USA) and Merck Co. (Darmstadt, Germany). Melting points were determined using Fisher-Johns apparatus (Fisher Scientific, Schwerte, Germany) and are uncorrected. The ${ }^{1} \mathrm{H}$-NMR and ${ }^{13} \mathrm{C}$-NMR spectra were recorded on a Bruker Avance spectrometer (Bruker BioSpin GmbH, Rheinstetten, Germany) using TMS as an internal standard. The IR spectra $(\mathrm{KBr})$ were obtained on a Perkin-Elmer 1725X FTIR spectrophotometer. Elemental analyses were performed on an AMZ 851 CHX analyzer (PG, Gdańsk, Poland) and the results were within $\pm 0.2 \%$ of the theoretical value. All the compounds were purified by flash chromatography (PuriFlash 430evo, Interchim, USA).

Synthesis of thiosemicarbazide derivatives (4-6)

Three derivatives of thiosemicarbazide: 1-benzoyl-4-(4bromophenyl)thiosemicarbazide (4), 4-(4-bromophenyl)1-[(2-chlorophenyl)carbonyl]thiosemicarbazide (5), and 4-(4-bromophenyl)-1-[(4-chlorophenyl)carbonyl]thiosemicarbazide (6) were synthesized according to the procedure described earlier (Plech et al., 2011a). Their spectral and physicochemical properties were consistent with (Li et al., 2001; Oruç et al., 2004).

Synthesis of 1,2,4-triazole derivatives (7-9)

Appropriate thiosemicarbazides (4-6) were dissolved in $2 \%$ solution of $\mathrm{NaOH}$. Next, the resulting solution was heated under reflux for $2 \mathrm{~h}$. After cooling, the reaction mixture was neutralized with $\mathrm{HCl}$. The precipitated product was filtered off, washed with distilled water, and recrystallized from $\mathrm{EtOH}$.

4-(4-Bromophenyl)-5-phenyl-2,4-dihydro-3H-1,2,4triazole-3-thione (7)

Yield: $87 \%$, CAS Registry Number: 162221-97-8.

4-(4-Bromophenyl)-5-(2-chlorophenyl)-2,4-dihydro-3H1,2,4-triazole-3-thione (8)

Yield: $83 \%$, m.p. $282-284{ }^{\circ} \mathrm{C},{ }^{1} \mathrm{H}-\mathrm{NMR} \quad(250 \mathrm{MHz})$ $\left(\right.$ DMSO- $\left.d_{6}\right) \delta$ (ppm): 7.08-7.76 (m, 8H, Ar-H), 14.03 (s, $1 \mathrm{H}, \mathrm{NH}$, exch. $\left.\mathrm{D}_{2} \mathrm{O}\right)$. IR $\left(\mathrm{KBr}, v, \mathrm{~cm}^{-1}\right): 3411,3045,1531$, 1331. Anal. Calc. for $\mathrm{C}_{14} \mathrm{H}_{9} \mathrm{BrClN}_{3} \mathrm{~S}(\%)$ : C 45.86, $\mathrm{H} 2.47$, N 11.46. Found: C 45.99, H 2.35, N 11.43. 
4-(4-Bromophenyl)-5-(4-chlorophenyl)-2,4-dihydro-3H1,2,4-triazole-3-thione (9)

Yield: $82 \%$, CAS Registry Number: 537017-82-6.

General procedure for the synthesis of Mannich bases (10-21)

$10 \mathrm{mmol}$ of the 1,2,4-triazole derivative (7-9) was dissolved (with heating) in $20 \mathrm{ml}$ of anhydrous ethanol and then equimolar amounts of appropriate secondary amine (diethylamine, pyrrolidine, piperidine, and morpholine) and formaldehyde solution (37\%) were added. The obtained mixture was stirred at room temperature for $30 \mathrm{~min}$. Next, $5 \mathrm{ml}$ of distilled water was added, the precipitate was filtered off, washed with distilled water, and recrystallized from ethanol.

4-(4-Bromophenyl)-2-[(diethylamino)methyl]-5-phenyl2,4-dihydro-3H-1,2,4-triazole-3-thione (10)

Yield: $78 \%$, m.p. $118-120{ }^{\circ} \mathrm{C},{ }^{1} \mathrm{H}-\mathrm{NMR} \quad(250 \mathrm{MHz})$ $\left(\mathrm{CDCl}_{3}\right) \delta(\mathrm{ppm}): 1.20\left(\mathrm{t}, 6 \mathrm{H}, 2 \times \mathrm{CH}_{3}, J=7.17 \mathrm{~Hz}\right)$, $2.90\left(\mathrm{q}, 4 \mathrm{H}, 2 \times \mathrm{CH}_{2}, J=7.18 \mathrm{~Hz}\right), 5.32\left(\mathrm{~s}, 2 \mathrm{H}, \mathrm{CH}_{2}\right)$, $7.18(\mathrm{~d}, 2 \mathrm{H}, \mathrm{Ar}-\mathrm{H}, J=8.69 \mathrm{~Hz}), 7.25-7.34(\mathrm{~m}, 5 \mathrm{H}, \mathrm{Ar}-$ $\mathrm{H}), 7.61(\mathrm{~d}, 2 \mathrm{H}, \mathrm{Ar}-\mathrm{H}, J=8.70 \mathrm{~Hz})$. IR $\left(\mathrm{KBr}, v, \mathrm{~cm}^{-1}\right)$ : 3065, 2931, 2796, 1612, 1520, 1331, 799. Anal. Calc. for $\mathrm{C}_{19} \mathrm{H}_{21} \mathrm{BrN}_{4} \mathrm{~S}(\%)$ : C 54.68, H 5.07, N 13.42. Found: C 54.60, H 5.02, N 13.53.

4-(4-Bromophenyl)-5-phenyl-2-(pyrrolidin-1-ylmethyl)2,4-dihydro-3H-1,2,4-triazole-3-thione (11)

Yield: $82 \%$, m.p. $142-143{ }^{\circ} \mathrm{C},{ }^{1} \mathrm{H}-\mathrm{NMR} \quad(250 \mathrm{MHz})$ $\left(\mathrm{CDCl}_{3}\right) \delta(\mathrm{ppm}): 1.75-1.83\left(\mathrm{~m}, 4 \mathrm{H}, 2 \times \mathrm{CH}_{2}\right), 2.99(\mathrm{t}$, $\left.4 \mathrm{H}, 2 \times \mathrm{CH}_{2}, J=6.43 \mathrm{~Hz}\right), 5.34\left(\mathrm{~s}, 2 \mathrm{H}, \mathrm{CH}_{2}\right), 7.19$ (d, $2 \mathrm{H}, \mathrm{Ar}-\mathrm{H}, J=8.86 \mathrm{~Hz}), 7.25-7.33(\mathrm{~m}, 5 \mathrm{H}, \mathrm{Ar}-\mathrm{H}), 7.61$ (d, $2 \mathrm{H}, \mathrm{Ar}-\mathrm{H}, J=8.84 \mathrm{~Hz})$. IR $\left(\mathrm{KBr}, v, \mathrm{~cm}^{-1}\right): 3084$, 3008, 2915, 2868, 1584, 1513, 1323, 806. Anal. Calc. for $\mathrm{C}_{19} \mathrm{H}_{19} \mathrm{BrN}_{4} \mathrm{~S}$ (\%): C 54.94, H 4.61, N 13.49. Found: C 55.05, H 4.50, 13.50 .

4-(4-Bromophenyl)-5-phenyl-2-(piperidin-1-ylmethyl)2,4-dihydro-3H-1,2,4-triazole-3-thione (12)

Yield: $77 \%$, m.p. $122-123{ }^{\circ} \mathrm{C},{ }^{1} \mathrm{H}-\mathrm{NMR} \quad(250 \mathrm{MHz})$ $\left(\mathrm{CDCl}_{3}\right) \delta(\mathrm{ppm}): 1.44-1.68\left(\mathrm{~m}, 6 \mathrm{H}, 3 \times \mathrm{CH}_{2}\right), 2.87(\mathrm{t}$, $\left.4 \mathrm{H}, 2 \times \mathrm{CH}_{2}, J=5.40 \mathrm{~Hz}\right), 5.25\left(\mathrm{~s}, 2 \mathrm{H}, \mathrm{CH}_{2}\right), 7.19$ (d, $2 \mathrm{H}, \mathrm{Ar}-\mathrm{H}, J=8.90 \mathrm{~Hz}), 7.24-7.35(\mathrm{~m}, 5 \mathrm{H}, \mathrm{Ar}-\mathrm{H}), 7.61$ $(\mathrm{d}, 2 \mathrm{H}, \mathrm{Ar}-\mathrm{H}, J=8.90 \mathrm{~Hz})$. IR $\left(\mathrm{KBr}, v, \mathrm{~cm}^{-1}\right): 3110$, 2918, 2785, 1603, 1519, 1342, 808. Anal. Calc. for $\mathrm{C}_{20} \mathrm{H}_{21} \mathrm{BrN}_{4} \mathrm{~S}$ (\%): C 55.94, $\mathrm{H}$ 4.93, N 13.05. Found: $\mathrm{C}$ 56.00, H 4.90, N 13.17.
4-(4-Bromophenyl)-2-(morpholin-4-ylmethyl)-5-phenyl2,4-dihydro-3H-1,2,4-triazole-3-thione (13)

Yield: $83 \%$, m.p. $\quad{ }^{146-147}{ }^{\circ} \mathrm{C},{ }^{1} \mathrm{H}-\mathrm{NMR} \quad(250 \mathrm{MHz})$ $\left(\mathrm{CDCl}_{3}\right) \delta(\mathrm{ppm}): 2.95\left(\mathrm{t}, 4 \mathrm{H}, 2 \times \mathrm{CH}_{2}, J=4.26 \mathrm{~Hz}\right)$, $3.76\left(\mathrm{t}, 4 \mathrm{H}, 2 \times \mathrm{CH}_{2}, J=4.26 \mathrm{~Hz}\right), 5.26\left(\mathrm{~s}, 2 \mathrm{H}, \mathrm{CH}_{2}\right)$, $7.18(\mathrm{~d}, 2 \mathrm{H}, \mathrm{Ar}-\mathrm{H}, J=8.80 \mathrm{~Hz}), 7.24-7.35(\mathrm{~m}, 5 \mathrm{H}, \mathrm{Ar}-$ H), $7.62(\mathrm{~d}, 2 \mathrm{H}, \mathrm{Ar}-\mathrm{H}, J=8.81 \mathrm{~Hz})$. IR $\left(\mathrm{KBr}, v, \mathrm{~cm}^{-1}\right)$ : 3074, 3021, 2961, 2831, 1574, 1512, 1328, 786. Anal. Calc. for $\mathrm{C}_{19} \mathrm{H}_{19} \mathrm{BrN}_{4} \mathrm{OS}(\%)$ : C 52.90, H 4.44, N 12.99. Found: C 52.98, H 4.56, N 13.05.

4-(4-Bromophenyl)-5-(2-chlorophenyl)-2-

[(diethylamino)methyl]-2,4-dihydro-3H-1,2,4-triazole-3thione (14)

Yield: $79 \%$, m.p. $172-173{ }^{\circ} \mathrm{C},{ }^{1} \mathrm{H}-\mathrm{NMR} \quad(250 \mathrm{MHz})$ $\left(\mathrm{CDCl}_{3}\right) \delta(\mathrm{ppm}): 1.20\left(\mathrm{t}, 6 \mathrm{H}, 2 \times \mathrm{CH}_{3}, J=7.55 \mathrm{~Hz}\right)$, $2.90\left(\mathrm{q}, 4 \mathrm{H}, 2 \times \mathrm{CH}_{2}, J=7.55 \mathrm{~Hz}\right), 5.30\left(\mathrm{~s}, 2 \mathrm{H}, \mathrm{CH}_{2}\right)$, $7.17(\mathrm{~d}, 2 \mathrm{H}, \mathrm{Ar}-\mathrm{H}, J=8.89 \mathrm{~Hz}), 7.22-7.32(\mathrm{~m}, 4 \mathrm{H}, \mathrm{Ar}-$ $\mathrm{H}), 7.62(\mathrm{~d}, 2 \mathrm{H}, \mathrm{Ar}-\mathrm{H}, J=8.90 \mathrm{~Hz}) . \mathrm{IR}\left(\mathrm{KBr}, v, \mathrm{~cm}^{-1}\right)$ : 3030, 2986, 2832, 1603, 1541, 1341, 813. Anal. Calc. for $\mathrm{C}_{19} \mathrm{H}_{20} \mathrm{BrClN}_{4} \mathrm{~S}(\%)$ : C 50.51, H 4.46, N 12.40. Found: C 50.41, H 4.38, N 12.29.

4-(4-Bromophenyl)-5-(2-chlorophenyl)-2-(pyrrolidin-1ylmethyl)-2,4-dihydro-3H-1,2,4-triazole-3-thione (15)

Yield: $84 \%$, m.p. $143-145{ }^{\circ} \mathrm{C},{ }^{1} \mathrm{H}-\mathrm{NMR} \quad(250 \mathrm{MHz})$ $\left(\mathrm{CDCl}_{3}\right) \delta(\mathrm{ppm}): 1.76-1.83\left(\mathrm{~m}, 4 \mathrm{H}, 2 \times \mathrm{CH}_{2}\right), 2.96(\mathrm{t}$, $\left.4 \mathrm{H}, 2 \times \mathrm{CH}_{2}, J=6.40 \mathrm{~Hz}\right), 5.32\left(\mathrm{~s}, 2 \mathrm{H}, \mathrm{CH}_{2}\right), 7.17(\mathrm{~d}$, $2 \mathrm{H}, \mathrm{Ar}-\mathrm{H}, J=8.75 \mathrm{~Hz}), 7.22-7.30(\mathrm{~m}, 4 \mathrm{H}, \mathrm{Ar}-\mathrm{H}), 7.63$ (d, $2 \mathrm{H}, \mathrm{Ar}-\mathrm{H}, J=8.75 \mathrm{~Hz})$. IR $\left(\mathrm{KBr}, v, \mathrm{~cm}^{-1}\right): 3099$, 2956, 2825, 1589, 1530, 1327, 802. Anal. Calc. for $\mathrm{C}_{19} \mathrm{H}_{18} \mathrm{BrClN}_{4} \mathrm{~S}(\%)$ : C 50.73, H 4.03, N 12.46. Found: C 50.66, H 4.12, N 12.45 .

4-(4-Bromophenyl)-5-(2-chlorophenyl)-2-(piperidin-1ylmethyl)-2,4-dihydro-3H-1,2,4-triazole-3-thione (16)

Yield: $80 \%$, m.p. $\quad{ }^{180-181 ~}{ }^{\circ} \mathrm{C},{ }^{1} \mathrm{H}-\mathrm{NMR} \quad(250 \mathrm{MHz})$ $\left(\mathrm{CDCl}_{3}\right) \delta(\mathrm{ppm}): 1.36-1.69\left(\mathrm{~m}, 6 \mathrm{H}, 3 \times \mathrm{CH}_{2}\right), 2.85(\mathrm{t}, 4 \mathrm{H}$, $\left.2 \times \mathrm{CH}_{2}, J=5.40 \mathrm{~Hz}\right), 5.22\left(\mathrm{~s}, 2 \mathrm{H}, \mathrm{CH}_{2}\right), 7.18(\mathrm{~d}, 2 \mathrm{H}, \mathrm{Ar}-$ $\mathrm{H}, J=8.71 \mathrm{~Hz}), 7.23-7.34(\mathrm{~m}, 4 \mathrm{H}, \mathrm{Ar}-\mathrm{H}), 7.63$ (d, 2H, Ar$\mathrm{H}, J=8.70 \mathrm{~Hz})$. IR $\left(\mathrm{KBr}, v, \mathrm{~cm}^{-1}\right): 3062,2985,2800$, 1594, 1526, 1342, 784. Anal. Calc. for $\mathrm{C}_{20} \mathrm{H}_{20} \mathrm{BrClN}_{4} \mathrm{~S}(\%)$ : C 51.79, H 4.35, N 12.08. Found: C 51.90, H 4.35, N 12.00.

4-(4-Bromophenyl)-5-(2-chlorophenyl)-2-(morpholin-4ylmethyl)-2,4-dihydro-3H-1,2,4-triazole-3-thione (17)

Yield: $76 \%$, m.p. $174-175{ }^{\circ} \mathrm{C},{ }^{1} \mathrm{H}-\mathrm{NMR} \quad(250 \mathrm{MHz})$ $\left(\mathrm{CDCl}_{3}\right) \delta$ (ppm): $2.91\left(\mathrm{t}, 4 \mathrm{H}, 2 \times \mathrm{CH}_{2}, J=4.75 \mathrm{~Hz}\right)$, 
$3.72\left(\mathrm{t}, 4 \mathrm{H}, 2 \times \mathrm{CH}_{2}, J=4.75 \mathrm{~Hz}\right), 5.23\left(\mathrm{~s}, 2 \mathrm{H}, \mathrm{CH}_{2}\right)$, $7.17(\mathrm{~d}, 2 \mathrm{H}, \mathrm{Ar}-\mathrm{H}, J=8.81 \mathrm{~Hz}), 7.23-7.34(\mathrm{~m}, 4 \mathrm{H}, \mathrm{Ar}-$ $\mathrm{H}), 7.64(\mathrm{~d}, 2 \mathrm{H}, \mathrm{Ar}-\mathrm{H}, J=8.81 \mathrm{~Hz})$. IR $\left(\mathrm{KBr}, v, \mathrm{~cm}^{-1}\right)$ : 3037, 2903, 2785, 1600, 1521, 1328, 806. Anal. Calc. for $\mathrm{C}_{19} \mathrm{H}_{18} \mathrm{BrClN}_{4} \mathrm{OS}(\%)$ : C 48.99, H 3.90, N 12.03. Found: C 49.11, H 3.84, N 12.17.

4-(4-Bromophenyl)-5-(4-chlorophenyl)-2-

[(diethylamino)methyl]-2,4-dihydro-3H-1,2,4-triazole-

3-thione (18)

Yield: $82 \%$, m.p. $175-176{ }^{\circ} \mathrm{C},{ }^{1} \mathrm{H}-\mathrm{NMR} \quad(250 \mathrm{MHz})$ $\left(\mathrm{CDCl}_{3}\right) \delta(\mathrm{ppm}): 1.20\left(\mathrm{t}, 6 \mathrm{H}, 2 \times \mathrm{CH}_{3}, J=7.24 \mathrm{~Hz}\right)$, $2.90\left(\mathrm{q}, 4 \mathrm{H}, 2 \times \mathrm{CH}_{2}, J=7.24 \mathrm{~Hz}\right), 5.30\left(\mathrm{~s}, 2 \mathrm{H}, \mathrm{CH}_{2}\right)$, $7.17(\mathrm{~d}, 2 \mathrm{H}, \mathrm{Ar}-\mathrm{H}, J=8.63 \mathrm{~Hz}), 7.22-7.33(\mathrm{~m}, 4 \mathrm{H}, \mathrm{Ar}-$ H), $7.62(\mathrm{~d}, 2 \mathrm{H}, \mathrm{Ar}-\mathrm{H}, J=8.63 \mathrm{~Hz})$. IR $\left(\mathrm{KBr}, v, \mathrm{~cm}^{-1}\right)$ : 3088, 3009, 2917, 2826, 1589, 1526, 1319, 778. Anal. Calc. for $\mathrm{C}_{19} \mathrm{H}_{20} \mathrm{BrClN}_{4} \mathrm{~S}(\%)$ : C 50.51, H 4.46, N 12.40. Found: C 50.43, H 4.52, N 12.41.

4-(4-Bromophenyl)-5-(4-chlorophenyl)-2-(pyrrolidin-1ylmethyl)-2,4-dihydro-3H-1,2,4-triazole-3-thione (19)

Yield: $87 \%$, m.p. $143-144{ }^{\circ} \mathrm{C},{ }^{1} \mathrm{H}-\mathrm{NMR} \quad(250 \mathrm{MHz})$ $\left(\mathrm{CDCl}_{3}\right) \delta(\mathrm{ppm}): 1.76-1.84\left(\mathrm{~m}, 4 \mathrm{H}, 2 \times \mathrm{CH}_{2}\right), 2.97(\mathrm{t}$, $\left.4 \mathrm{H}, 2 \times \mathrm{CH}_{2}, J=6.10 \mathrm{~Hz}\right), 5.32\left(\mathrm{~s}, 2 \mathrm{H}, \mathrm{CH}_{2}\right), 7.18(\mathrm{~d}$, $2 \mathrm{H}, \mathrm{Ar}-\mathrm{H}, J=8.76 \mathrm{~Hz}), 7.23-7.34(\mathrm{~m}, 4 \mathrm{H}, \mathrm{Ar}-\mathrm{H}), 7.64$ (d, $2 \mathrm{H}, \mathrm{Ar}-\mathrm{H}, J=8.76 \mathrm{~Hz})$. IR $\left(\mathrm{KBr}, v, \mathrm{~cm}^{-1}\right)$ : 3040, 2921, 2787, 1609, 1541, 1333, 783. Anal. Calc. for $\mathrm{C}_{19} \mathrm{H}_{18} \mathrm{BrClN}_{4} \mathrm{~S}$ (\%): C 50.73, H 4.03, N 12.46. Found: C 50.62, H 3.92, N 12.52 .

4-(4-Bromophenyl)-5-(4-chlorophenyl)-2-(piperidin-1ylmethyl)-2,4-dihydro-3H-1,2,4-triazole-3-thione (20)

Yield: $76 \%$, m.p. ${ }^{180-182}{ }^{\circ} \mathrm{C},{ }^{1} \mathrm{H}-\mathrm{NMR} \quad(250 \mathrm{MHz})$ $\left(\mathrm{CDCl}_{3}\right) \delta(\mathrm{ppm}): 1.36-1.68\left(\mathrm{~m}, 6 \mathrm{H}, 3 \times \mathrm{CH}_{2}\right), 2.85(\mathrm{t}$, $\left.4 \mathrm{H}, 2 \times \mathrm{CH}_{2}, J=5.62 \mathrm{~Hz}\right), 5.22\left(\mathrm{~s}, 2 \mathrm{H}, \mathrm{CH}_{2}\right), 7.18(\mathrm{~d}$, $2 \mathrm{H}, \mathrm{Ar}-\mathrm{H}, J=8.74 \mathrm{~Hz}), 7.23-7.31(\mathrm{~m}, 4 \mathrm{H}, \mathrm{Ar}-\mathrm{H}), 7.63$ $(\mathrm{d}, 2 \mathrm{H}, \mathrm{Ar}-\mathrm{H}, J=8.72 \mathrm{~Hz}) .{ }^{13} \mathrm{C}-\mathrm{NMR}(90 \mathrm{MHz})\left(\mathrm{CDCl}_{3}\right)$ $\delta$ (ppm): 23.81, 25.91, 51.82, 71.09, 123.64, 124.10, 129.11, 129.87, 130.02, 133.27, 134.45, 137.27, 148.18, 170.64. IR (KBr, $\left.v, \mathrm{~cm}^{-1}\right): 3085,2882,2790,1600,1531$, 1323, 809. Anal. Calc. for $\mathrm{C}_{20} \mathrm{H}_{20} \mathrm{BrClN}_{4} \mathrm{~S}(\%)$ : C 51.79, $\mathrm{H}$ 4.35, N 12.08. Found: C 51.86, H 4.32, N 12.18.

4-(4-Bromophenyl)-5-(4-chlorophenyl)-2-(morpholin-4ylmethyl)-2,4-dihydro-3H-1,2,4-triazole-3-thione (21)

Yield: $80 \%$, m.p. ${ }^{177-178}{ }^{\circ} \mathrm{C},{ }^{1} \mathrm{H}-\mathrm{NMR} \quad(250 \mathrm{MHz})$ $\left(\mathrm{CDCl}_{3}\right) \delta(\mathrm{ppm}): 2.91\left(\mathrm{t}, 4 \mathrm{H}, 2 \times \mathrm{CH}_{2}, J=4.73 \mathrm{~Hz}\right), 3.73$ (t, $\left.4 \mathrm{H}, 2 \times \mathrm{CH}_{2}, J=4.70 \mathrm{~Hz}\right), 5.23\left(\mathrm{~s}, 2 \mathrm{H}, \mathrm{CH}_{2}\right), 7.17$ (d, $2 \mathrm{H}, \mathrm{Ar}-\mathrm{H}, J=8.70 \mathrm{~Hz}), 7.25-7.34(\mathrm{~m}, 4 \mathrm{H}, \mathrm{Ar}-\mathrm{H}), 7.64(\mathrm{~d}$,
$2 \mathrm{H}, \mathrm{Ar}-\mathrm{H}, J=8.70 \mathrm{~Hz})$. IR $\left(\mathrm{KBr}, v, \mathrm{~cm}^{-1}\right): 3074,3033$, 2951, 2856, 1603, 1541, 1318, 798. Anal. Calc. for $\mathrm{C}_{19} \mathrm{H}_{18} \mathrm{BrClN}_{4} \mathrm{OS}(\%)$ : C 48.99, H 3.90, N 12.03. Found: C 49.10, H 3.97, N 12.00 .

Antibacterial screening

Tested microorganism: S. aureus ATCC 25923, S. aureus Microbank 14001 (MRSA), Staphylococcus epidermidis ATCC 12228, B. subtilis ATCC 6633, B. cereus ATCC 10876, M. luteus ATCC 10240, E. coli ATCC 25922, K. pneumoniae ATCC 13883, P. mirabilis ATCC 12453, and $P$. aeruginosa ATCC 9027. Preliminary antibacterial in vitro potency of the tested compounds was screened using the agar dilution method on the basis of the growth inhibition on the Mueller-Hinton agar to which the tested compounds at concentration $1,000 \mu \mathrm{g} \mathrm{ml}^{-1}$ were added. The plates were poured on the day of testing. $10 \mu \mathrm{l}$ of each bacterial suspension was put onto Mueller-Hinton agar containing the tested compounds; medium without the compounds was used as a control. The plates were incubated at $37{ }^{\circ} \mathrm{C}$ for $18 \mathrm{~h}$. Then the in vitro antibacterial activity of the compounds with inhibitory effect was determined by broth microdilution method. Ampicillin, cefuroxime, and vancomycin were used as control antimicrobial agents. The microbial suspensions were prepared in sterile saline with an optical density of 0.5 McFarland standard$150 \times 10^{6} \mathrm{CFU} \mathrm{ml}{ }^{-1}$ (CFU—colony forming unit). All stock solutions of the tested compounds were dissolved in DMSO. Mueller-Hinton broth was used with a series of twofold dilutions of the tested substances in the range of final concentrations from 3.91 to $1,000 \mu \mathrm{g} \mathrm{ml}^{-1}$. Minimum inhibitory concentration (MIC) and minimum bactericidal concentration (MBC) are given in $\mu \mathrm{g} \mathrm{ml}^{-1}$ (CLSI 2008).

Open Access This article is distributed under the terms of the Creative Commons Attribution License which permits any use, distribution, and reproduction in any medium, provided the original author(s) and the source are credited.

\section{References}

Almajan GL, Barbuceanu SF, Almajan ER, Draghici C, Saramet G (2009) Synthesis, characterization and antibacterial activity of some triazole Mannich bases carrying diphenylsulfone moieties. Eur J Med Chem 44:3083-3089

Ashok M, Holla BS, Poojary B (2007) Convenient one pot synthesis and antimicrobial evaluation of some new Mannich bases carrying 4-methylthiobenzyl moiety. Eur J Med Chem 42: 1095-1101

Bayrak H, Demirbas A, Karaoglu SA, Demirbas N (2009a) Synthesis of some new 1,2,4-triazoles, their Mannich and Schiff bases and evaluation of their antimicrobial activities. Eur J Med Chem 44:1057-1066 
Bayrak H, Demirbas A, Demirbas N, Karaoglu SA (2009b) Synthesis of some new 1,2,4-triazoles starting from isonicotinic acid hydrazide and evaluation of their antimicrobial activities. Eur J Med Chem 44:4362-4366

CLSI (2008) Performance standards for antimicrobial susceptibility testing; eighteenth international supplement. CLSI document M7-MIC. Clinical Laboratory Standards Institute, Wayne

Eswaran S, Adhikari AV, Shetty NS (2009) Synthesis and antimicrobial activities of novel quinoline derivatives carrying 1,2,4triazole moiety. Eur J Med Chem 44:4637-4647

Isloor AM, Kalluraya B, Shetty P (2009) Regioselective reaction: synthesis, characterization and pharmacological studies of some new Mannich bases derived from 1,2,4-triazoles. Eur J Med Chem 44:3784-3787

Li JP, Luo QF, Wang YL, Wang H (2001) An efficient solid-state method for the preparation of acylthiosemicarbazides. Synth Commun 31:1793-1797

Oruç EE, Rollas S, Kandemirli F, Shvets N, Dimoglo AS (2004) 1,3,4-Thiadiazole derivatives. Synthesis, structure elucidation and strucuture-antituberculosis activity relationship investigation. J Med Chem 47:6760-6767

Plech T, Wujec M, Siwek A, Kosikowska U, Malm A (2011a) Synthesis and antimicrobial activity of thiosemicarbazides, $s$ triazoles and their Mannich bases bearing 3-chlorophenyl moiety. Eur J Med Chem 46:241-248
Plech T, Wujec M, Kaproń B, Kosikowska U, Malm A (2011b) Synthesis and antibacterial activity of some novel N2-hydroxymethyl and N2-aminomethyl derivatives of 4-aryl-5-(3-chlorophenyl)-2,4-dihydro-3H-1,2,4-triazole-3-thione. Heteroat Chem 22:737-743

Rolain JM, Parola P, Cornaglia G (2010) New Delhi metallo-betalactamase (NDM-1): towards a new pandemia? Clin Microbiol Infect 16:1699-1701

Shafiee A, Sayadi A, Roozbahani MH, Foroumadi A, Kamal F (2002) Synthesis and in vitro antimicrobial evaluation of 5-(1-methyl-5nitro-2-imidazolyl)-4H-1,2,4-triazoles. Arch Pharm Pharm Med Chem 10:495-499

Turan-Zitouni G, Kaplancıklı ZA, Yıldız MT, Chevallet P, Kaya D (2005) Synthesis and antimicrobial activity of 4-phenyl/cyclohexyl-5-(1-phenoxyethyl)-3-[ $N$-(2-thiazolyl)acetamido]-thio4H-1,24-triazole derivatives. Eur J Med Chem 40:607-613

Wujec M, Kosikowska U, Paneth P, Malm A (2007) Reaction of hydrazide of (tetrazol-5-yl)acetic acid with isothiocyanates and antimicrobial investigations of newly-obtained compounds. Heterocycles 71:2617-2626

Yong D, Toleman MA, Giske CG, Cho HS, Sundman K, Lee K, Walsh $\mathrm{T}$ (2009) Characterization of a new metallo- $\beta$-lactamase gene, blaNDM-1, and a novel erythromycin esterase gene carried on a unique genetic structure in Klebsiella pneumoniae sequence type 14 from India. Antimicrob Agents Chemother 53:5046-5054 